\title{
Experience with Sorafenib in Patients with Advanced Hepatocellular Carcinoma Treated at the National Cancer Institute of Panama (Sorafen Study)
}

\author{
Ronald Jahir Dominguez-Molina ${ }^{1,}$, Jose Pinto-Llerena ${ }^{2, *}$ \\ ${ }^{1}$ Medical Oncology Fellow, Department of Medical Oncology, National Cancer Institute, Panama City, Panama \\ ${ }^{2}$ Medical Oncology, Unit of Gastrointestinal Tumors, Department of Medical Oncology, National Cancer Institute, Panama City, Panama
}

Email address:

josepintollerena@gmail.com (J. Pinto-Llerena)

${ }^{*}$ Corresponding author

\section{To cite this article:}

Ronald Jahir Dominguez-Molina, Jose Pinto-Llerena. Experience with Sorafenib in Patients with Advanced Hepatocellular Carcinoma Treated at the National Cancer Institute of Panama (Sorafen Study). International Journal of Clinical Oncology and Cancer Research.

Vol. 6, No. 1, 2021, pp. 17-21. doi: 10.11648/j.ijcocr.20210601.13

Received: January 5, 2021; Accepted: January 18, 2021; Published: January 25, 2021

\begin{abstract}
Sorafenib is an oral multi-kinase inhibitor that increases survival and delays tumor progression in patients with advanced hepatocarcinoma (HCC). This study aimed to determine the progression-free survival (PFS) and overall survival (OS) and to analyze OS-related factors in this population between 2014 and 2019. We performed a retrospective review of patients with advanced HCC who were treated with sorafenib at the National Cancer Institute of Panama (Instituto Oncologico Nacional de Panama - IONP) from January 2014 to December 2019. The data were collected from electronic health records of the IONP. In total, 77 patients with a mean age of 65 years were analyzed. Sixty-three percent of the patients were men, and most of them had an Eastern Cooperative Oncology Group (ECOG) performance status of 1 and had not undergone any prior treatment. Forty-four percent patients had Child-Pugh Class A. The most frequent progression site was the liver (27\%), followed by the lungs. Mean PFS was 1 month, and mean OS was 3 months. The clinical benefit was $39 \%$ and Overall response rate was 3,9\%; for those with stable disease, it was 35,1\%, and 3,9\% showed a partial response. More encouraging results were obtained from patients with higher functional statuses (ECOG status 0-1) and who were in milder stages of liver disease (Child-Pugh Class A). The most common adverse events were fatigue, hand-foot syndrome, nausea and vomiting, and arterial hypertension. The survival results assessed in our institution for patients with advanced hepatocellular carcinoma treated with sorafenib are below of those published in the literature; however, after selecting the cases with ECOG 0-1 and Child A stage, our results were more in line with those of the international literature, being the most important prognostic factors in our patients. Fatigue and hand-foot syndrome were the most common adverse events. The key to the success of this therapy lies in an adequate selection of patients.
\end{abstract}

Keywords: Hepatocarcinoma (HCC), Sorafenib, Progression-free Survival (PFS), Overall Survival (OS)

\section{Introduction}

Hepatocellular carcinoma (HCC) is the most common primary malignancy of the liver $[1,2]$. The fifth most common cancer among men and the ninth most common cancer among women $(7.5 \%$ and $3.4 \%$ of all cancers, respectively). HCC is also the fourth leading cause of cancer-related death worldwide and resulted in approximately 781,631 deaths ( $8.2 \%$ of all deaths) in 2018 and accounts for $4.7 \%$ of all cancers, with 841,080 new cases (4.7\% of all cancers) [3]. In Panama, HCC is the seventh most frequent cancer and the sixth deadliest, with a $6.2 \%$ mortality rate [4].

Sorafenib has been considered the standard of care for patients with unresectable, advanced HCC since 2007 and numerous studies have investigated the role of markers involved in the angiogenesis [5-7].

In a Phase 3 trial, llovet et al. demonstrated that sorafenib, an oral multi-kinase inhibitor, delayed tumor progression and increased survival among patients with advanced HCC [8]. Sorafenib inhibits the activity of several tyrosine kinases involved in angiogenesis and tumor progression, including 
vascular endothelial growth factor receptor 2/3 (VEGFR-2/3), platelet-derived growth factor receptor (PDGF-R) and others [9-11].

In 2008, the Sorafenib Hepatocellular Carcinoma Assessment Randomized Protocol (SHARP) trial demonstrated the efficacy and safety of sorafenib. In this Phase 3, placebo-controlled, randomized clinical trial with 602 patients, the survival of patients treated with sorafenib increased by nearly three months (10.7 vs. 7.9 months, $\mathrm{p}<0.001)$, and there were also differences in radiological progression in favor of patients treated with the drug (5.5 vs. 2.8 months, $\mathrm{p}<0.001$ ) without significant differences in symptomatic progression $(\mathrm{p}<0.77)[8,12]$.

A recent pooled analysis of two-phase III randomized trials showed that the neutrophil-to-lymphocyte ratio, etiology and extra-hepatic spread are predictive factors of response to sorafenib but did not identify any predictive biological markers [7, 13].

Wada et al. analyzed 86 patients with radiological progression after treatment with sorafenib, including 47 who continued treatment despite radiological progression, and 39 who underwent a second line of treatment. PFS was higher in the group that continued sorafenib (2.6 months) than in the group that discontinued treatment (1.4 months, $\mathrm{p}<0.01)$. Patients who continued treatment with sorafenib had a longer mean OS (12.9 months) than those who discontinued treatment (4.5 months, $\mathrm{p}<0.01$ ). Accordingly, multivariate analysis showed that continuing treatment with sorafenib was an independent predictor of survival (14). In contrast, Nakano et al. conducted a study with a similar design, but came to an opposite conclusion. In a cohort of 141 patients who progressed radiologically despite their treatment with sorafenib, 58 continued treatment, 70 received another type of treatment, and 13 received no treatment. The cohort that received another treatment had a mean survival of over 12.2 months, whereas the patients who continued treatment with sorafenib had a mean survival of 6.1 months $(p<0.001)[15,16]$.

The primary endpoint of this study was to determine the PFS and OS of patients with advanced HCC who were treated with sorafenib. The secondary endpoint was to analyze clinical and demographic factors related to OS among patients with advanced HCC who were treated with sorafenib at the IONP.

\section{Materials and Methods}

\subsection{Patient Selection}

Data were retrospectively collected from patients with advanced HCC treated with sorafenib between January 2014 and December 2019. The data were retrieved from electronic health records and from the Oncofarmis database of the IONP, with prior consent of the head of the medical oncology department and head of teaching.

All patients diagnosed with advanced HCC by histopathological or radiological confirmation who were treated with sorafenib were included in this study.
The patients who died within 15 days of starting treatment due to unrelated complications were excluded from this study.

We defined PFS as the time, in months, from treatment onset to disease progression. We defined OS as the time, in months, from treatment onset to death or study completion.

\subsection{Follow-Up}

Patients visited the IONP for a follow-up consultation with the attending medical oncologist every 4 weeks, and their responses to treatment were evaluated by tomography after 3 months, applying the Response Evaluation Criteria in Solid Tumors (RECIST) 1.1.

\subsection{Statistical Analysis}

Data were collected and analyzed using IBM SPSS Statistics, version 24, license 1989, 2017. Patient characteristics were reported using frequency and descriptive analysis. The Kaplan-Meier method was used to analyze PFS and OS. We used non-parametric statistics to compare relationships between variables.

\section{Results}

The records of 77 patients with HCC who were treated with sorafenib from January 2014 to December 2019 were revisited. The mean age was 65 years, $66,2 \%$ patients were men, and $33,8 \%$ patients were women. Most patients had Eastern Cooperative Oncology Group (ECOG) performance status scores of $0-1(76,6 \%)$, and had not received prior treatment $(63,6 \%)$. In total, $41,6 . \%$ patients were classified as Child-Pugh Class A. The most frequent progression sites were the liver $(28,6 \%)$ and the lungs $(20 \%)$ (Table 1$)$.

Table 1. Population Characteristics $n=77$.

\begin{tabular}{lll}
\hline Characteristic & Number & Percentage (\%) \\
\hline Sex & & \\
F & 26 & 33,8 \\
M & 51 & 66,2 \\
Age & & \\
Mean & $65 \pm 13,3$ years & \\
ECOG & & \\
0-1 & 59 & 76,6 \\
$\geq-2$ & 18 & 23,4 \\
Prior Therapy & & \\
None & 49 & 63,6 \\
Chemoembolization & 25 & 32,5 \\
Chemoembolization + RFA & 2 & 2,6 \\
Surgery & 1 & 1,3 \\
Child-Pugh Score & & \\
A & 32 & 41,6 \\
B & 26 & 33,8 \\
C & 3 & 3,9 \\
Unknown & 16 & 20,8 \\
Progression Sites & & \\
Liver & 22 & 28,6 \\
Liver and Lung & 7 & 9,1 \\
Lung & 9 & 11.7 \\
Other sites & 39 & 50,6 \\
Total Patients & 77 & 100 \\
\hline
\end{tabular}

RFA: Radiofrequency ablation 


\subsection{Progression-Free Survival}

The median PFS time was 1 month (0.3-1.6 months) (Figure 1). Exploratory analysis by functional status (ECOG 0-1) and liver disease severity (Child-Pugh A) showed significant differences in PFS among ECOG levels; patients with median ECOG 0-1 had a PFS of 5 months, and the patients with an ECOG $\geq 2$ had a PFS of 0.1 month $(\mathrm{p}=0.001)$. Univariate analysis showed that having ECOG levels 0-1, receiving treatment before sorafenib, and being in Child-Pugh Class A are prognostic factors for PFS (Table 2).

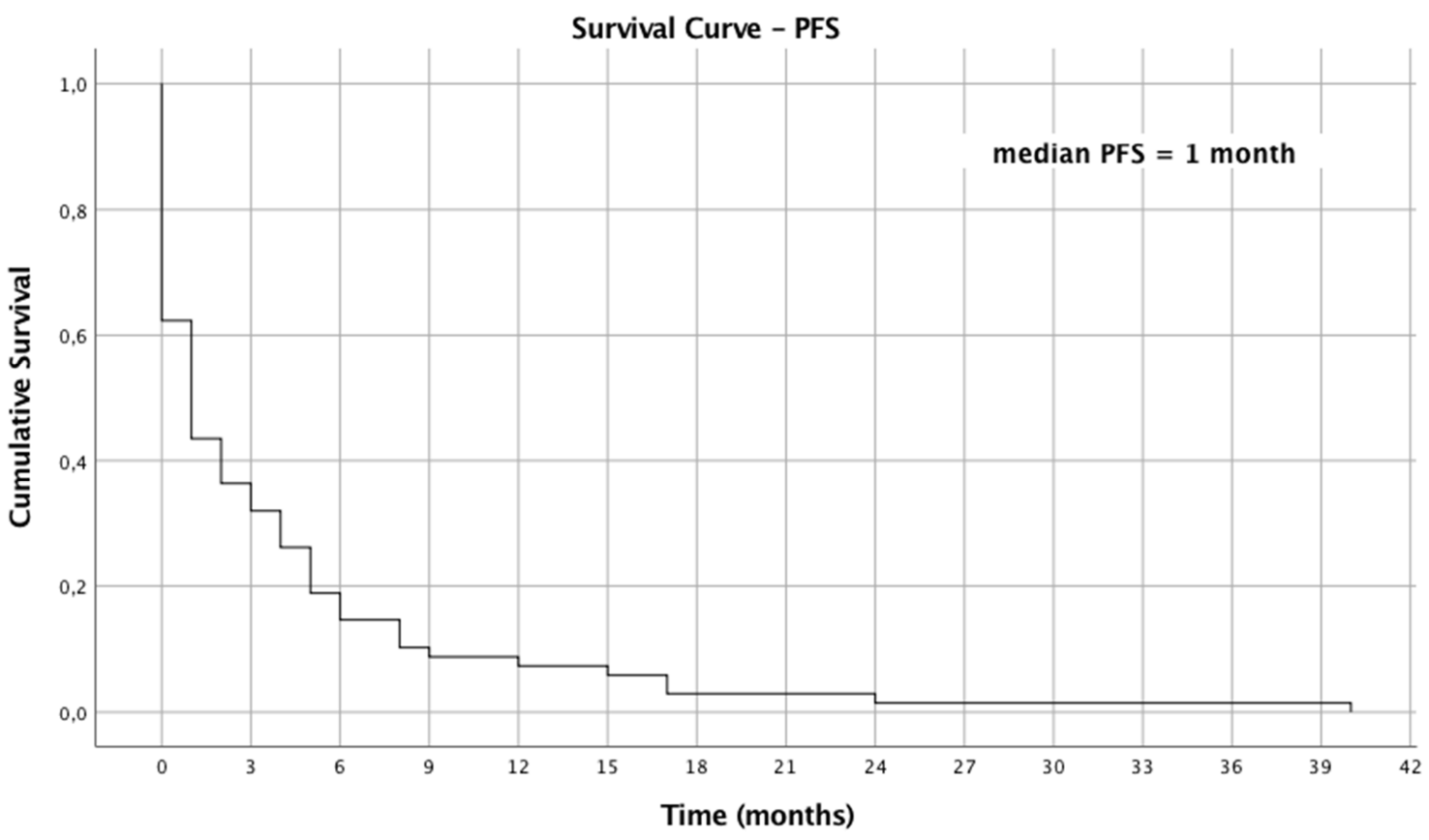

Figure 1. Progression-Free Survival (PFS).

\subsection{Overall Survival}

The median OS was 3 months (1.6-4.3 months) (Figure 2). Exploratory analysis by functional status (ECOG level 0-1) and liver disease severity (Child-Pugh Class A) showed significant differences in OS between patients with an ECOG level $\geq 2$, who had a median OS of 1 month, and patients with an ECOG level 0-1, who had a median OS of 9 months $(\mathrm{p}=0.001)$.

Univariate analysis showed that ECOG levels $0-1$, being in Child-Pugh Class A, and normal alpha-fetoprotein levels at treatment onset were favorable prognostic factors for OS (Table 2).

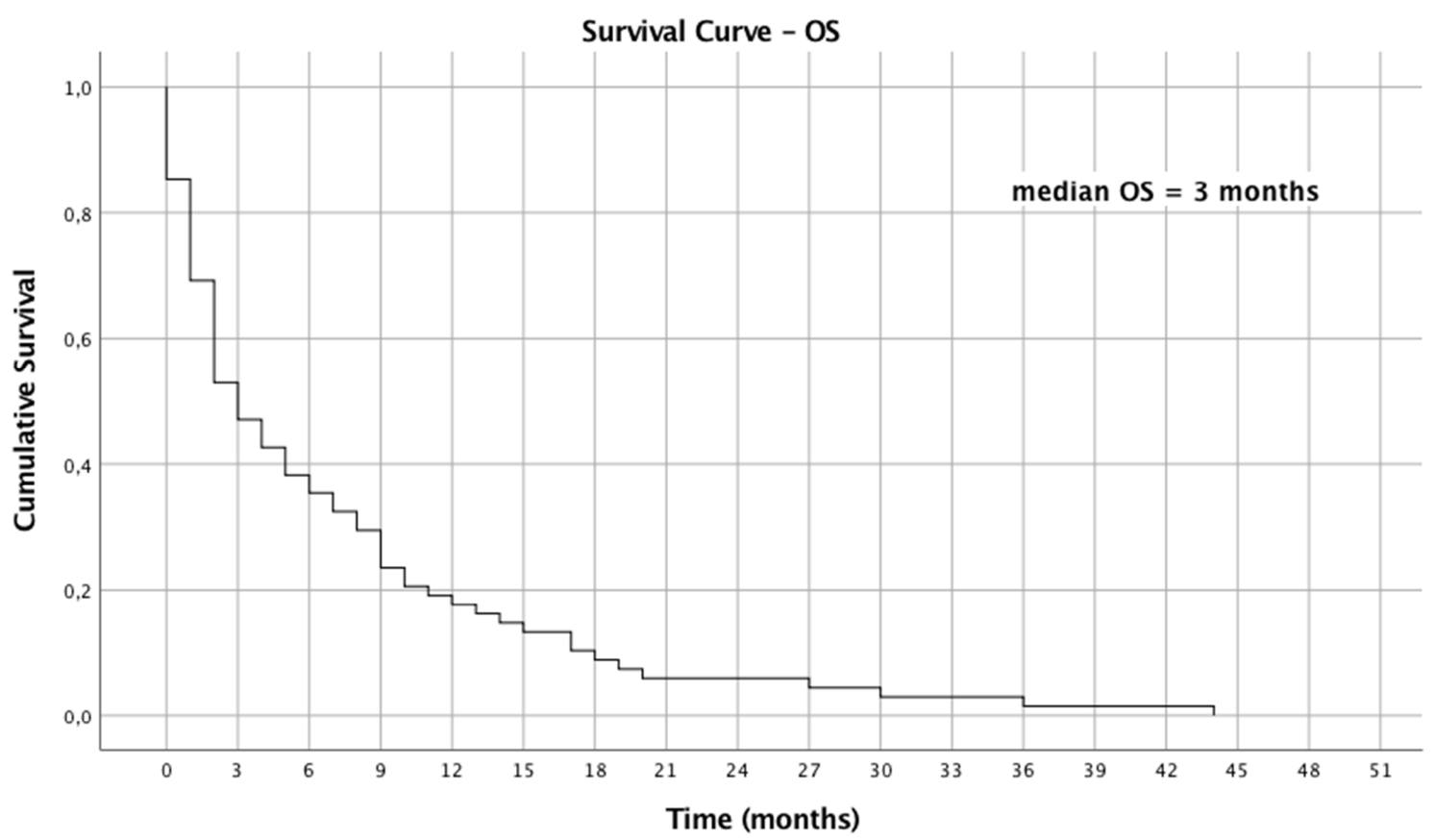

Figure 2. Overall Survival (OS). 
Table 2. PFS- and OS-related prognostic factors.

\begin{tabular}{lll}
\hline Parameter & Progression-Free Survival H. R. & Overall Survival H. R. \\
\hline ECOG & 7.410 & 8.426 \\
$0-1$ vs $\geq 2$ & $(\mathrm{p}=0.006)$ & $(\mathrm{p}=0.004)$ \\
Child-Pugh Score & 26.744 & $17.372(\mathrm{p}=0.001)$ \\
A vs B and C & $(\mathrm{p} \leqq 0.001)$ & \\
Prior Treatment & 4.282 & 2.251 \\
Yes vs No & $(\mathrm{p} \leqq 0.039)$ & $(\mathrm{p}=0.134)$ \\
Hepatitis $B$ & 0.647 & 0.002 \\
Yes vs No & $(\mathrm{p}=0.421)$ & $(\mathrm{p}=0.962)$ \\
Initial AFP & 5.621 & 7.446 \\
Normal vs Positive and $\geq 100000$ & $(\mathrm{p} \leqq 0.060)$ & $(\mathrm{p} \leqq 0.024)$ \\
Age & 2.868 & 1.205 \\
$>65$ or $<65$ years & $(\mathrm{p}=0.090)$ & $(\mathrm{p}=0.272)$ \\
Sex & 0.543 & 1.701 \\
F vs M & $(\mathrm{p}=0.461)$ & $(\mathrm{p}=0.192)$ \\
Cirrhosis & 2.568 & 2.841 \\
Yes vs No & $(\mathrm{p}=0.277)$ & $(\mathrm{p}=0.242)$ \\
\hline
\end{tabular}

\subsection{Response to Treatment}

The clinical benefit was $39,0 \%$, for those with stable disease it was $35,1 \%$, and $3,9 \%$ showed a partial response (Table 3). More encouraging results were obtained from patients with higher functional statuses (ECOG0-1) and who were in milder stages of liver disease.

Table 3. Response Rate.

\begin{tabular}{ll}
\hline Type of Response & Response Rate (\%) \\
\hline No Response & 61,0 \\
Stable Disease & 35,1 \\
Partial & 3,9 \\
Overall Response Rate & 3,9 \\
\hline
\end{tabular}

\subsection{Adverse Events}

The most common adverse events were fatigue (17\%), hand-foot syndrome $(17 \%)$, nausea and vomiting $(6.1 \%)$, and arterial hypertension (6.1\%). Most events were classified as grade 1 , that is, well tolerated (Table 4).

Table 4. Adverse Events related to Sorafenib

\begin{tabular}{ll}
\hline Adverse Event & Frequency (\%) \\
\hline None & $15(18 \%)$ \\
Fatigue & $14(17 \%)$ \\
Hand Foot Syndrome & $14(17 \%)$ \\
Vomiting and nausea & $5(6.1 \%)$ \\
Arterial Hypertension & $5(6.1 \%)$ \\
Transaminitis & $4(4.9 \%)$ \\
Thrombocytopenia & $3(3.7 \%)$ \\
Diarrhea & $2(2.4 \%)$ \\
Mucositis & $2(2.4 \%)$ \\
\hline
\end{tabular}

\section{Discussion}

This is the first study that shows our institution's experiences with the use of sorafenib to manage patients with HCC.

The overall results (mPFS $=1$ month and $\mathrm{mOS}=3$ months) differed from those reported in other papers; this is possibly due to the large proportion of patients in our study who had low functional statuses (ECOG level $\geq 2$ ) and were in more advanced stages of liver disease (Child-Pugh Classes B and C). Exploratory analysis by functional status (ECOG levels 0-1) and liver disease severity (Child-Pugh Class A) showed significant differences in PFS, with patients with ECOG levels of $0-1$ reaching a mPFS of 5 months and an mOS of 9 months. These values are similar to those obtained in the SHARP trial.

The following PFS- and OS-related prognostic factors in patients treated with sorafenib were found in our study: Child-Pugh Scale score, prior treatment, ECOG level, and AFP levels. Prior treatment improved PFS outcomes but had no impact on OS. The Gideon study, an observational registry of sorafenib use in Child-Pugh subgroups, showed that patients who were in Child-Pugh Class A have higher OS [17].

In the SHARP trial, AFP plasma levels $>200 \mathrm{ng} / \mathrm{ml}$ were a negative prognostic marker [8], and their early decline is a predictive biomarker, as shown by Shao et al. [18]. Responders were compared with non-responders with significantly improved ORR (33\% vs. 8\%, p=0.037) and DCR ( $83 \%$ vs. $35 \%, p=0.002)$. In addition, the mPFS was 7.5 months vs. 1.9 months $(\mathrm{p}=0.001)$, and the mOS was 15.3 months vs. 4.1 months $(\mathrm{p}=0.019)$ for responders and non-responders, respectively $[8,19,20]$.

Notwithstanding the limitations of our retrospective, single-center study and with a small number of patients, the results of our institution, which treats approximately $95 \%$ of the malignant tumors of Panama, are nonetheless an extraordinary contribution to the research on sorafenib in the region.

\section{Conclusions}

The survival results assessed in our institution for patients with advanced hepatocellular carcinoma treated with sorafenib are below of those published in the literature; however, after selecting the cases with ECOG 0-1 and Child A stage, our results were more in line with those of the international literature, being the most important prognostic factors in our patients. Fatigue and hand-foot syndrome were the most common adverse events. The key to the success of this therapy lies in an adequate selection of patients. 


\section{Conflict of Interests}

The authors declare that there is no conflict of interest in this study.

\section{Acknowledgements}

The authors thank Omar Castillo Fernandez, MD, Chief of the Medical Oncology Department, National Cancer Institute of Panama (Instituto Oncologico Nacional Panama - IONP), for his advice in preparing the protocol and reviewing the data, the members of the unit of Gastrointestinal Tumors, IONP, for their consulting and project review, and Rosana Arango, MD, third-year Resident in Internal Medicine at the Saint Thomas Hospital (Hospital Santo Tomás - HST), Panama, for her help with the statistical analysis and data collection.

\section{References}

[1] Ghouri Y, Mian I, Rowe J. Review of hepatocellular carcinoma Epidemiology, etiology, and carcinogenesis. J Carcinog. 2017; 16 (1): 1 .

[2] Rashed WM, Kandeil MAM, Mahmoud MO, Ezzat S Hepatocellular Carcinoma (HCC) in Egypt: A comprehensive overview. J Egypt Natl Cancer Inst. 2020; 32 (1): 5.

[3] Bray F, Ferlay J, Soerjomataram I, Siegel RL, Torre LA, Jemal A. Global cancer statistics 2018: GLOBOCAN estimates of incidence and mortality worldwide for 36 cancers in 185 countries. CA Cancer J Clin. 2018; 68 (6): 394-424.

[4] boletin_2016_rncp.pdf [Internet]. [cited 16 de enero de 2021]. Disponible http://minsa.b-cdn.net/sites/default/files/general/boletin_2016 _ncp.pdf.

[5] Furuse J. Sorafenib for the treatment of unresectable hepatocellular carcinoma. Biol Targets Ther. 2008; 779.

[6] Raoul J-L, Adhoute X, Penaranda G, Perrier H, Castellani P, Oules V, et al. Sorafenib: Experience and Better Manage-ment of Side Effects Improve Overall Survival in Hepatocellular Carcinoma Patients: A Real-Life Retrospective Analysis. Liver Cancer. 2019; 8 (6): 457-67.

[7] Marisi G, Cucchetti A, Ulivi P, Canale M, Cabibbo G, Solaini L, et al. Ten years of sorafenib in hepatocellular carcinoma: Are there any predictive and/or prognostic markers? World J Gastroenterol. 2018; 24 (36): 4152-63.

[8] Llovet JM, Ricci S, Mazzaferro V, Hilgard P, Gane E, Blanc J-F, et al. Sorafenib in Advanced Hepatocellular Carcinoma. N Engl J Med. 2008; 359 (4): 378-90.

[9] Esteban-Villarrubia J, Soto-Castillo JJ, Pozas J, San Román-Gil M, Orejana-Martín I, Torres-Jiménez J, et al.
Tyrosine Kinase Receptors in Oncology. Int J Mol Sci. 2020; 21 (22): 8529.

[10] Qin S, Li A, Yi M, Yu S, Zhang M, Wu K. Recent advances on anti-angiogenesis receptor tyrosine kinase inhibitors in cancer therapy. J Hematol OncolJ Hematol Oncol. 2019; 12 (1): 27.

[11] Gotink KJ, Verheul HMW. Anti-angiogenic tyrosine kinase inhibitors: what is their mechanism of action? Angiogenesis. 2010; 13 (1): 1-14.

[12] Bruix J, Raoul J-L, Sherman M, Mazzaferro V, Bolondi L, Craxi A, et al. Efficacy and safety of sorafenib in patients with advanced hepatocellular carcinoma: Subanalyses of a phase III trial. J Hepatol. 2012; 57 (4): 821-9.

[13] Bruix J, Cheng A-L, Meinhardt G, Nakajima K, De Sanctis Y, Llovet J. Prognostic factors and predictors of sorafenib benefit in patients with hepatocellular carcinoma: Analysis of two-phase III studies. J Hepatol. 2017; 67 (5): 999-1008.

[14] Wada Y, Takami Y, Tateishi M, Ryu T, Mikagi K, Saitsu H. The Efficacy of Continued Sorafenib Treatment after Radiologic Confirmation of Progressive Disease in Patients with Advanced Hepatocellular Carcinoma. Huo T-I, editor. PLOS ONE. 2016; 11 (1): e0146456.

[15] Kurume Liver Cancer Study Group of Japan, Nakano M, Tanaka M, Kuromatsu R, Nagamatsu H, Satani M, et al. Alternative treatments in advanced hepatocellular carcinoma patients with progressive disease after sorafenib treatment: a prospective multicenter cohort study. Oncotarget. 2016; 7 (39): 64400-9.

[16] Toyoda H, Kumada T, Kaneoka Y, Osaki Y, Kimura T, Arimoto A, et al. Prognostic value of pretreatment levels of tumor markers for hepatocellular carcinoma on survival after curative treatment of patients with HCC. J Hepatol. 2008; 49 (2): 223-32.

[17] Marrero JA, Kudo M, Venook AP, Ye S-L, Bronowicki J-P, Chen X-P, et al. Observational registry of sorafenib use in clinical practice across Child-Pugh subgroups: The GIDEON study. J Hepatol. 2016; 65 (6): 1140-7.

[18] Shao Y-Y, Lin Z-Z, Hsu C, Shen Y-C, Hsu C-H, Cheng A-L. Early alpha-fetoprotein response predicts treatment efficacy of antiangiogenic systemic therapy in patients with advanced hepatocellular carcinoma. Cancer. 2010; 116 (19): 4590-6.

[19] Brunetti O, Gnoni A, Licchetta A, Longo V, Calabrese A, Argentiero A, et al. Predictive and Prognostic Factors in HCC Patients Treated with Sorafenib [Internet]. MEDICINE \& PHARMACOLOGY; 2019 sep. Disponible in: https://www.preprints.org/manuscript/201909.0071/v1.

[20] Apostolidis L, Pfeiffenberger J, Gotthardt D, Radeleff B, Mehrabi A, Schemmer P, et al. Survival of Hepatocellular Carcinoma Patients Treated with Sorafenib beyond Progression. Gastrointest Tumors. 2018; 5 (1-2): 38-46. 